\title{
Existence \& Distribution of Government Associated STD Clinics - United States, 2017
}

\author{
Beth E. Meyerson PhD,a,b Alissa Davis PhD,c,d Laura T. Haderxhanaj PhD, ,a,b Michael A. Sayegh PhD,e \\ Megan K. Simmons PhD, ${ }^{a, b}$ Gurprit Multani MPH,, ${ }^{a, b}$ Lindsey Naeyaert MPH, ${ }^{\text {a,b }}$ Audra Meador MPH ${ }^{a, b}$
}

a Indiana University School of Public Health-Bloomington, Department of Applied Health Science, Indiana University, Bloomington, Indiana USA Rural Center for AIDS/STD Prevention, Indiana University, Bloomington, Indiana USA

HIV Center for Clinical and Behavioral Studies, Columbia University Medical Center and New York Psychiatric Institute, New York, NY USA

d Social Intervention Group, Columbia University School of Social Work, New York, NY 10027, USA

e Indiana University School of Public Health-Bloomington, Department of Epidemiology and Biostatistics, Indiana University, Bloomington, Indiana USA

Background: Sexually Transmitted Disease (STD) Clinics provide an important safety net for sexual health services. Attempts at national STD clinic studies have been limited by volunteer, purposeful or limited geographic sampling because a national list of STD clinics does not exist in the U.S.

Study Objective: To establish the existence and distribution of U.S. government-associated STD clinics with reference to selected community characteristics likely associated with need for STD services.

Methods: A two-phased multi-level internet-based search concluding in October 2017 identified STD clinics in U.S. states and the District of Columbia. Data included clinic name, address, clinic contact information, 340B funding status, and type of clinic. Secondary, county-level data for comparison included rates of chlamydia and HIV, teen births, uninsurance and unemployment, high school graduation, primary care physician ratio, healthcare costs, median household income and percent living in rural areas. Binary logistic regression investigated difference between counties without and with STD clinics, and among types of clinics.

\begin{tabular}{|c|c|c|c|c|}
\hline & $\begin{array}{c}\text { County } \\
\text { has an } \\
\text { STD } \\
\text { Clinic }\end{array}$ & Mean & $\begin{array}{c}\text { Std. } \\
\text { Deviation }\end{array}$ & $\begin{array}{l}\text { Values with } \\
p<.001 \\
t()=\text { df }\end{array}$ \\
\hline \multirow{2}{*}{$\begin{array}{l}\text { Chlamydia rate } \\
(\mathbf{N}=2910)\end{array}$} & Yes & 367.88 & 186.33 & $T(-15.08)=2254.9$ \\
\hline & No & 269.62 & 153.77 & \\
\hline \multirow{2}{*}{$\begin{array}{l}\text { Percent uninsured } \\
<65 \text { yrs. of age } \\
(\mathrm{N}=3 \mid 25)\end{array}$} & Yes & 0.14 & 0.05 & $T(3.37)=1830.01$ \\
\hline & No & 0.15 & 0.06 & \\
\hline \multirow{2}{*}{$\begin{array}{l}\text { High School } \\
\text { Graduation rate } \\
(\mathrm{N}=267 \mathrm{I})\end{array}$} & Yes & 0.85 & 0.08 & $T(I I .47)=2669$ \\
\hline & No & 0.89 & 0.08 & \\
\hline \multirow{2}{*}{$\begin{array}{l}\text { Percent Unemployed } \\
>16 \text { yrs. of age } \\
(\mathrm{N}=3 \text { I 35) }\end{array}$} & Yes & 0.06 & 0.02 & $T(-11.10)=3133$ \\
\hline & No & 0.05 & 0.02 & \\
\hline \multirow{2}{*}{$\begin{array}{l}\text { Social associations } \\
\text { rate per I0K pop } \\
(\mathrm{N}=3 \mid 36)\end{array}$} & Yes & 12.70 & 5.62 & $T(10.78)=1619.13$ \\
\hline & No & 15.82 & 8.68 & \\
\hline \multirow{2}{*}{$\begin{array}{l}\text { HIV prevalence rate } \\
\text { ( } N=2338)\end{array}$} & yes & 185.18 & 169.24 & $T(-10.74)=1519.44$ \\
\hline & No & 118.32 & 115.48 & \\
\hline \multirow{2}{*}{$\begin{array}{l}\text { Percent living in rural } \\
\text { area }(\mathrm{N}=3 \mid 36)\end{array}$} & yes & 0.52 & 0.31 & $T(15.75)=2417.76$ \\
\hline & No & 0.70 & 0.29 & \\
\hline
\end{tabular}

Conclusions: The resulting national clinic list will permit future, more comprehensive national studies of clinic services, organizational adaptation to external policy and funding environments, and clinic survival over time. Special attention should be given to clinic coverage in rural areas.

\section{Next Steps:}

$\checkmark$ Validate national list

$\checkmark$ Investigate and classify levels of STI services per national tiering

$\checkmark$ Measure clinic responsiveness by tier to community needs

$\checkmark$ Observe survival of these public clinics over time and through periods of health reform

$\checkmark$ Translate and communicate findings for program and policy reform

Key References

Meyerson BE, Davis A, Haderxhanaj LT, Sayegh MA, Simmons MK, Multani G Naeyaert L, Meador A. Existence and distribution of government associated STD clinics - United States, 2017 (in review).

Landry DJ, Forrest JD. Public health departments providing sexually transmitted disease services. Family Planning Perspectives 1996; 28:261-266.

Pathela P, Klingler EJ, Guerry SL, Bernstein KT, Kerani RP, Llata L, Mark HD, Tabidze I, Rietmeijer CA, SSuN Working Group. Sexually transmitted infection clinics as safety net providers: Exploring the role of categorical sexually transmitted infection clinics in an era of health care reform. Sex Transm Disease $2015 ; 42(5), 286-293$

- Clinics were classified into 10 clinic types. Over half (62\%) were Local Health Department Clinics. All others: Family Planning Clinics (17.1\%), Community Health Centers (7.8\%), School-based (3.6\%), State Health Departments (3.4\%), Hospital sponsored (2.2\%), AIDS Service Organizations (2.1\%), University sponsored (1.2\%), correctional settings $(0.6 \%)$, Other $(0.1 \%)$. AIDS/STD PREVENTION

- STD clinics tended to exist where the needs were greatest, with the exception of populations living in rural areas.
INDIANA UNIVERSITY

School of Public Health Bloomington 